\title{
A generalized solid state kinetic expression for reaction interface-controlled reactivity
}

\author{
Heinrich Badenhorst ${ }^{\star}$, Brian Rand, Walter Focke \\ SARChl Chair in Carbon Materials and Technology, Department of Chemical Engineering, University \\ of Pretoria, Lynwood Road, Pretoria, 0002, South Africa \\ *Corresponding author. Tel.: +27 12420 4173; Fax: +27 124202516. \\ Email address: heinrich.badenhorst@up.ac.za
}

\begin{abstract}
The ICTAC-recommended approach was used to characterize the gasification of high-purity, highly crystalline, large natural graphite flakes in oxygen. The average activation energy was found to be $157.7 \pm 4.2 \mathrm{~kJ} \mathrm{~mol}^{-1}$. The graphite properties and the simple gasification reaction taking place make this an ideal material for the study of reaction interface-controlled reactivity. Based on simple structural and geometrical observations, it was expected that the conversion function would be that of a shrinking disc. However, the experimental conversion function exhibited a behaviour which could not be linked to any of the commonly established reaction models.

A factor contributing to this disconnection is the use of an arbitrary scaling procedure in classic solid state kinetics. A more integrated approach has recently been proposed in the literature with the potential for reconciling disparate models into a single comprehensive scheme. A generalization of the classic solid state kinetic expressions for interface-controlled reactivity is proposed which fits into the integrated approach. It is based on fundamental considerations for the subset of reactions in which reactivity is controlled by the reaction interface alone. The fundamental nature of the approach yields an expression for which all the variables are directly measurable, without any assumptions regarding the conversion function.

The generalized conversion function will always start at a value of one, making interpretation and direct comparison of any active surface area (ASA) progression possible. Visual observations indicate the growth of defect structures within the macro graphite flakes, leading to an increase in ASA. This leads to a behaviour resembling nucleation and growth, despite the interface-controlled reaction taking place on a disc-shaped solid. The random nature of the oxidized flake microstructure makes it difficult to develop representative analytical models for this behaviour.
\end{abstract}

Keywords: Kinetics, Graphite oxidation, Reaction model, Reaction interface

\section{Introduction}

The solid state kinetic approach has been widely applied to the thermal decomposition of solids [1]. These reactions are fundamentally different from classic homogeneous reactions. For example, the concept of concentration is usually not applicable to the solid reactant. Crystalline solids contain defective or boundary regions which are more susceptible to chemical change than identical material 
elsewhere within the solid. This leads to heterogeneous reaction behaviour in which the kinetics are usually not determined by the amount of reactant available.

These reactions frequently occur only within a thin contact zone, the reaction interface, which advances into the remaining reactant. The zone undergoing reaction may be the total surface area, as in the case of a sphere, or a specific surface region, as in the case of a disc reacting only at the edges, or even, in the case of a porous solid, the pore surface area. For the purpose of this investigation, all three will be referred to as the active surface area (ASA). Thus the reaction rate is fundamentally determined by geometrical variations of the ASA, under the assumption that the surface is homogeneous and the material is isotropic.

However, in most solid state decompositions other complex phenomena are also occurring, such as melting, sintering, etc. The situation is further complicated by the presence of different crystal defects and impurities. The solid state approach has also been applied to other solid transitions, such as crystallization or crystallographic transitions, and autocatalytic reactions, such as the thermal decomposition of potassium permanganate [2]. These reactions are subject to other rate-controlling factors such as nucleation and growth, or in some cases where a product barrier layer is formed, diffusion through this layer.

Graphite is a solid reactant with a well-known crystal structure that reacts directly with gaseous oxygen to produce gaseous carbon dioxide or carbon monoxide, depending on conditions. In general, graphite materials can have a very high purity and secondary reactions are limited with virtually no ash generation. Therefore graphite represents an ideal starting material for investigating the reaction at a solid interface, where only gaseous reaction products are formed and the reaction rate is governed solely by the reactive interface.

Due to its industrial significance, a large number of the studies on graphite are focused on bulk synthetic graphite materials. Natural graphite flakes are expected to have far larger crystallites and superior graphitic order compared with synthetic graphite [3]. Furthermore, due to the manufacturing process, synthetic graphite has a very complex microstructure and porosity [4]. These properties make this type of graphite very difficult to model easily from the perspective of solid state kinetics. Instead it was decided here to focus on the simpler, flake-type natural graphite. These particles represent a solid reactant, with no porosity, which should be easily characterized by the solid state kinetic approach.

The approach recommended by the ICTAC (International Confederation for Thermal Analysis and Calorimetry) [5] was applied to the natural graphite to determine its kinetic parameters. However, these flakes exhibit a conversion behaviour that is difficult to reconcile with the classic solid state kinetic models. A modified form of the solid state kinetic rate equation is proposed which is more general in its applicability to solid reactants where reactivity is governed solely by the progression of the reaction interface. Finally, the observed conversion function is represented analytically, in terms of directly observed microstructural effects.

\section{Theory \\ 2.1 Classic solid state kinetic expression for reaction interface-controlled reactivity}


When the graphite flakes are examined under a scanning electron microscope (SEM), they appear to have a simple structure. As can be seen from Fig. 1, they are roughly disc-shaped, with some irregularities along the edges.

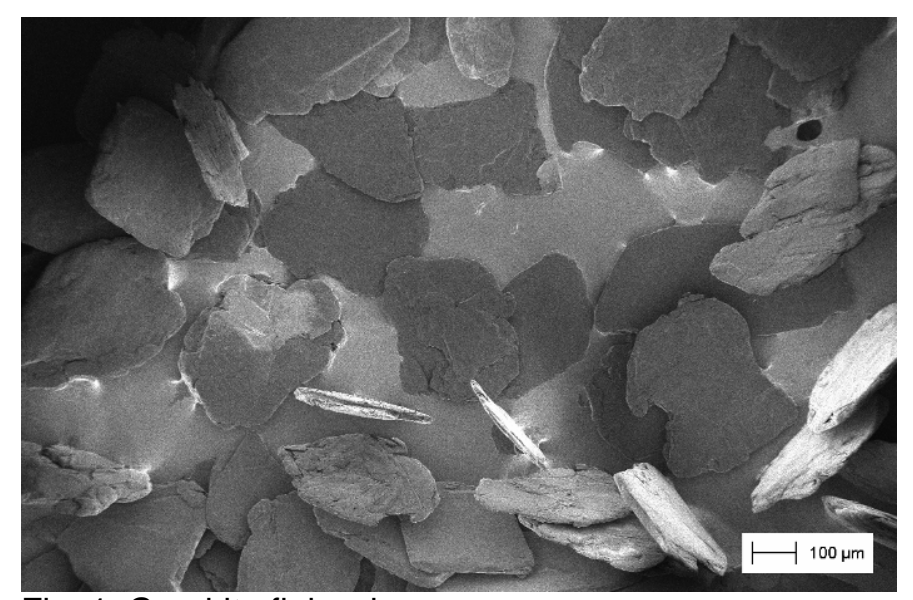

Fig. 1: Graphite flake shapes

The flakes are solid with a very high aspect ratio. The structure of ideal graphite is layered, planes of hexagons forming a honeycomb structure, due to the $\mathrm{sp}^{2}$ hybridisation of the carbon atoms. Adjacent layers are weakly bonded to each other by van der Waals forces [4]. This can be seen schematically in Fig. 2.

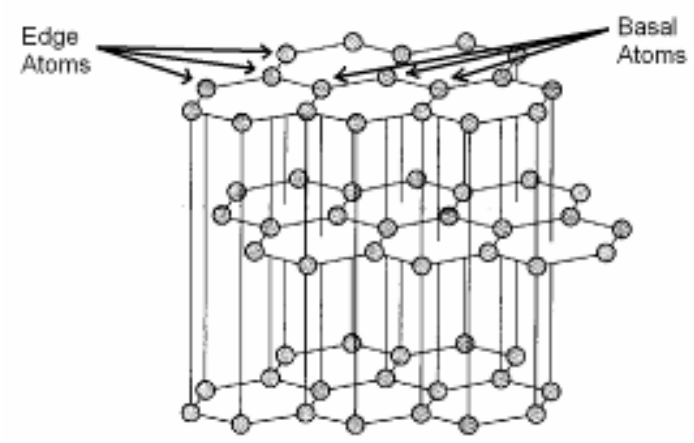

Fig. 2: Ideal graphite structure

When the particles are examined more closely, as in Fig. 3, the layered structure becomes clear and the edges can be seen to be fairly uniform and flat without porosity. This is consistent with expectations for highly crystalline graphite flakes. The slight roughness which is apparent on the upper flake surface is a very thin layer of damaged structure which is created during beneficiation. On the whole, the flake is homogenous and non-porous. 


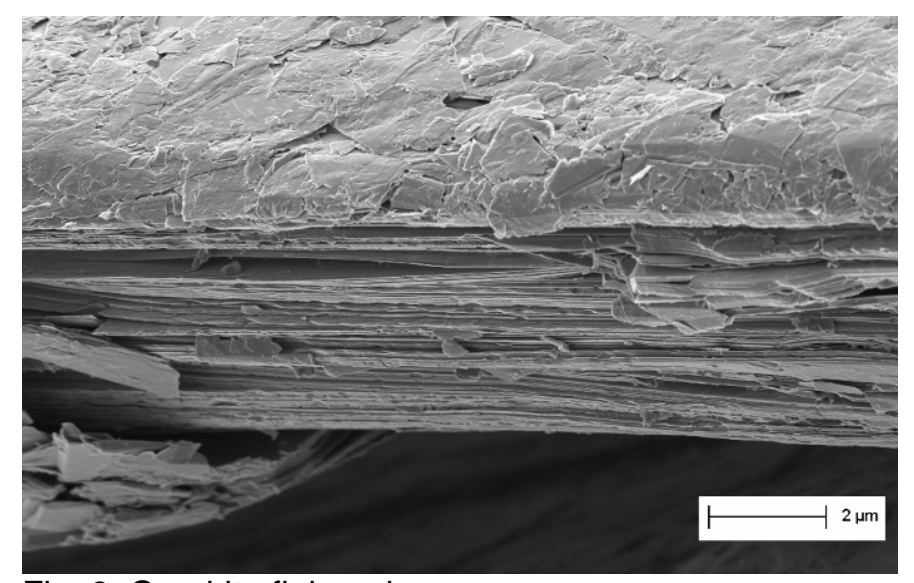

Fig. 3: Graphite flake edge

Natural graphite flakes are generally considered more consistently crystalline across the entire particle compared with synthetic materials. Due to the strong, inplane covalent bonding, the basal plane of graphite, i.e. the flat flake surface, is considered to be comparatively inert [6]. Thus attack by oxygen is only possible at the edge atoms shown in Fig. 2, except at extreme temperatures. Nucleation is instantaneous and the reaction proceeds simultaneously at all active sites. A very slight difference exists between the reactivity of armchair and zig-zag edge sites [7]. However, since a distribution of both sites is expected to be present, it may be assumed that a single, average reactivity prevails. Hence, the most applicable reaction model from the table of common models given by Vyazovkin et al. [5] would appear to be that of a contracting cylinder or disc, shown schematically in Fig. 4.

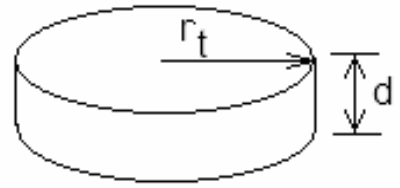

Fig. 4: Ideal natural graphite flake

This model is derived from the assumption that the reaction interface proceeds at a uniform velocity, $v$, towards the centre of the particle [8]. On an atomic level, this assumption is not strictly correct, since atoms will be removed at random from the surface and this will produce molecular irregularities on the surface. Statistically, these irregularities will be more likely to react and thus they will be preferentially removed, eventually leading to the development of a pseudo steady state. Thus on a macro scale it is assumed that the edge recession rate is a constant, averaged value and the reaction rate is governed only by the geometrical progression of the interface. The absolute value of the recession rate will naturally depend on the temperature. The classic solid state kinetic expression for such a reaction is given by:

$$
\begin{aligned}
& \frac{d \alpha}{d t}=k(T, P) f(\alpha) \\
& \text { with } \\
& k(T, P)=\frac{v(T)}{r_{0}} P_{02}^{m}
\end{aligned}
$$


and

$$
f(\alpha)=n(1-\alpha)^{\frac{n-1}{n}}
$$

where $r_{0}$ is the initial value of the "characteristic dimension" of the particle in question.

This expression is equally valid for cylinders where $n=2$ and spheres with $n=3$. The equation may also be applied to the theoretical case of an infinite flat plate, where $n=1$. For the carbon-oxygen reaction, the dependence on the gaseous reactant partial pressure has been shown to be a simple $m$-th order expression [9]. However, for the case of pure oxygen as reactant, this term reduces to a value of one. Typically, an Arrhenius equation is used for the temperature dependence [5], i.e.

$$
k(T)=k_{0} \exp \left(\frac{-E_{A}}{R T}\right)
$$

Any of the isoconversional methods recommended by ICTAC [5] may be used to determine the activation energy, $E_{A}$, independent of the conversion function associated with the reaction under consideration. Next, the conversion function may be obtained directly from the experimental data, isothermal or non-isothermal, by rearranging Eq. (1) with Eq. (4) substituted:

$$
k_{0} f(\alpha)=\frac{d \alpha}{d t} \exp \left(\frac{E_{A}}{R T}\right)
$$

The pre-exponential factor may now be arbitrarily chosen to scale the resulting curve to vary between zero and one [5]. This curve can then be compared against the scaled theoretical reaction model plots of known reaction mechanisms to identify the one that best fits the data. If such a mechanism can be found, the corresponding kinetic parameters of the model may be calculated - for example, the edge recession rate using Eq. (2) - if it is possible to define a "characteristic dimension".

This approach has several restrictive underlying assumptions as pointed out by Pijolat et al. [10]. Firstly, for the reaction under consideration here, the expressions for the geometry of the reaction interface are limited to the three cases mentioned earlier. As will be shown later, these are not adequate descriptions for the microstructural development observed in real particles. Secondly, the definition of "reaction rate" is not intuitive. For shapes where it is difficult, if not impossible, to define a "characteristic dimension", the interfacial velocity, $v$, cannot be experimentally determined, thus making comparison of samples from different origins, with potentially different reactivities, very difficult. Finally, as will be demonstrated, the arbitrary scaling applied when using Eq. (4) leads to difficulty in identifying, interpreting and comparing the experimentally observed conversion functions obtained for real samples.

\subsection{Generalized expression for reaction interface-controlled reactivity}

As mentioned earlier, the solid state kinetic approach has been applied to a wide range of complex reactions, with very disparate mechanisms controlling the reaction rate. The models representing these mechanisms were to a large degree developed 
independently, each with its own assumptions. They are largely empirical and based on observations of the resulting behaviour, rather than being directly linked to the solid reactant on a fundamental level. Unfortunately, this has led to a loss of the links between these mechanisms, as well as a loss of the ability to use physically observable phenomena to identify the mechanisms. This has inevitably resulted in the need to resort to curve fitting and an arbitrary scaling procedure [5], rather than linking the characteristics of the reactant during the reaction to a mechanism before establishing the reaction model. In order to re-establish the link between these models, it was suggested by Pijolat et al. [10] that a more formalized approach should be utilized to describe the solid state kinetics of solid-gas reactions. The authors proposed the general expression:

$$
\frac{d \alpha}{d t}=\phi\left(T, P_{i}\right) S_{m}(t, \ldots)
$$

In this expression, the $\phi\left(T, P_{i}\right)$ term or "aeric reactivity of growth", is related to the rate-determining step and only varies with the thermodynamic variables. The term $S_{m}(t, \ldots)$ or "space function" may be considered as a molar "active surface", at time $t$, corresponding to the growth process of one mole of reacting phase. This expression covers the cases of instantaneous nucleation and growth, nucleation and instantaneous growth, and all possibilities in between, together with the generation of a product phase.

Pijolat et al. [10] point out that for complex cases where the adsorption and desorption of multiple species on different types of active site are occurring, it may not be possible to separate the effect of temperature and partial pressure. This may explain why for such cases Arrhenius behaviour is not obtained, i.e. the activation energy, $E_{A}$, varies with the conversion, $\alpha$. In such cases it should rather be noted that an apparent activation energy, $E_{A p p}$, is actually being measured.

It is also stressed that the term $S_{m}(t, \ldots)$ can in some cases not be explicitly expressed as a function of conversion, e.g. when nucleation and growth are simultaneous. The generalized expression given by Eq. (6) may possibly be applied to the entire range of solid-gas reactions, acting as a first step towards the unification of the disparate mechanisms and as a starting point for deriving generalized expressions for the individual mechanisms. The expression can easily be linked to the reaction interface-controlled cases discussed in the previous section and the authors show that:

$$
k(T)=\frac{n V_{m A}}{r_{0}} \phi\left(T, P_{i}\right)
$$

in which case the $n$ term found in the classic expression for the conversion function, i.e. Eq. (3), has been moved into the kinetic term, i.e. Eq. (2). However, Eq. (7) holds no clear advantage over the classic expression given by Eq. (2) since it still depends on the definition of a "characteristic dimension", $r_{0}$, which limits its generality and applicability.

Both of these equations do, however, indicate that the pre-exponential factor, $k_{0}$, is not simply a kinetic constant but is intimately coupled to the geometry and ASA of the reactant under consideration. If an arbitrary scaling is applied when calculating $k_{0}$, this relationship is entirely lost. In general, $k_{0}$ is usually not clearly defined in the literature for kinetic analysis of reactions in powders. This may lead to incorrect conclusions when the results from different studies are compared or when $k_{0}$ is used for predictions or reactor modelling. There is a need to establish an expression that 
will fundamentally incorporate the kinetic parameters and the ASA, which will make possible to compare the results for two samples, both undergoing interfacecontrolled reactivity, but with different conversion functions and potentially different reactivities.

To overcome these shortcomings and in order to elucidate the fundamental nature of the pre-exponential factor, the oxidation of graphite may be used as a starting point. Consider the intrinsic reaction rate or atomic turnover frequency for this reaction:

$$
k^{I}=\frac{\text { number of carbon atoms reacted per } \mathrm{sec} \text { ond at time } t}{\text { number of carbon atoms available for reaction at time } t}
$$

The expression is given in terms of carbon but could just as easily be applied to any other solid reactant. This is the fundamental representation of the reaction rate, irrespective of the particle geometry and ASA progression. It is dependent only on the temperature. This value has been measured [11] during pit growth studies on highly ordered pyrolytic graphite (HOPG). One may assume, in accordance with Laine et al. [12], that the area occupied by a single carbon atom is $A_{C \text { ATOM }}=8.3 \AA^{2}=$ $0.83 \times 10^{-19} \mathrm{~m}^{2}$. Thus the denominator may be converted to ASA by multiplying by this value. It is important to note that this is again applicable to any reactive surface area, e.g. total surface area, pore area, etc. The numerator may be converted to mass by dividing by Avogadro's number $\left(N_{A}\right)$ and multiplying by the molecular mass of carbon $\left(M M_{C}\right)$. Thus:

$k^{A S A}=k^{I} \frac{M M_{C}}{N_{A} A_{C \text { АтоM }}}$ with units of $\left[\frac{\text { grams of carbon atoms reacted per } \mathrm{sec} \text { ond at time } t}{m^{2} \text { ASA at time } t}\right]$

The reaction rate may then be multiplied by the ASA (at any time) and divided by the initial mass to yield:

$$
\frac{d \alpha}{d t}=k^{A S A} \frac{A S A}{m_{0}} \text { with units of }\left[\frac{\text { grams of carbon reacted per } \mathrm{sec} \text { ond at time } t}{\text { grams of carbon present initially, time } t=0}\right]
$$

This is entirely consistent with the units of the reaction rate in Eq. (1) if one considers that:

$$
\frac{d \alpha}{d t}=\frac{-1}{m_{0}} \frac{d m_{t}}{d t}
$$

where $m_{0}$ is the initial mass and $m_{t}$ is the mass at time $t$.

Thus from a fundamental perspective the following solid state kinetic expression is consistent with the intrinsic reaction rate:

$$
\frac{d \alpha}{d t}=k^{I} \frac{M M_{C}}{N_{A} A_{C \text { ATOM }}} \frac{A S A}{m_{0}}=k^{A S A} \frac{A S A}{m_{0}}
$$

In accordance with Pijolat et al. [10], the rate expression should be split into a kinetic element depending only on the thermodynamic variables, and an active surface component. In Eq. (9), only one term has a temperature dependence, i.e. $k^{\prime}=$ $k^{\prime}(T)$, hence $k^{A S A}=k^{A S A}(T)$.

Consider a particle for which the progression of its reaction interface, i.e. the ASA development, can be exclusively stated to be a function of the dimensionless degree of conversion. In such a case, dividing this function by the initial ASA must necessarily yield a function that always starts at one, irrespective of the geometry and the type of ASA progression. Thus it makes sense that for all particles undergoing reaction solely at an interface, the ASA conversion function, $f^{A S A}(\alpha)$, can 
be normalized to a starting value of one by dividing by the initial ASA in the reaction rate expression. Thus the conversion function may be defined as:

$$
f^{A S A}(\alpha)=\frac{A S A}{A S A_{0}}
$$

and hence the general expression becomes:

$$
\frac{d \alpha}{d t}=\frac{A S A_{0}}{m_{0}} k^{A S A}(T) f^{A S A}(\alpha)
$$

It should be noted that for the purposes of this investigation, which was conducted in pure oxygen, the dependence on the gaseous reactant partial pressure reduces to one. Now the reaction rate constant $k^{A S A}(T)$ has a clear connection to the intrinsic reaction rate, and the ASA conversion function, $f^{A S A}(\alpha)$, has a clear definition: it is the normalized progression of the active surface area during the reaction. It can be shown (see Appendix A) that the classic expressions for a disc and a sphere are special cases of Eq. (11), which result when the following two conditions are met:

- The particle mass, at any time during the reaction, can be expressed in terms of a single "characteristic dimension".

- The change in mass of the particle, i.e. the mass of material reacted, is linearly proportional to the surface area undergoing reaction and the change in the characteristic dimension.

In which case one finds that:

$$
k^{A S A}(T)=\rho r_{0} k(T)=\rho v(T)
$$

And

$$
f^{A S A}(\alpha)=\frac{1}{n} f(\alpha)
$$

The same Arrhenius temperature dependence may be used:

$$
k^{A S A}(T)=k_{0}^{A S A} \exp \left(\frac{-E_{A}}{R T}\right)
$$

Comparison of Eq. (11) and Eq. (6) shows that the term $k^{A S A}(T)$ is equivalent to a specific case of the "aeric reactivity of growth", while the "space function" is given by $f^{A S A}(\alpha)^{*} A S A_{0} / m_{0}$. Thus Eq. (11) represents a specific case of the generalized expression given by Eq. (6) where the reaction rate is controlled by the interfacial reactivity alone. However, Eq. (11) is more generalized than the classic solid state kinetic expressions since it is applicable to any possible geometry with any conceivable ASA progression, without the need for the definition of a "characteristic dimension". It should be noted however that the term $k^{A S A}(T)$ is in fact $k^{A S A}(T, P)$, but as mentioned earlier, for graphite the pressure dependence is usually a simple $m$-th order expression, which reduces to one for experiments in pure oxygen.

As mentioned earlier, Hahn [11] found the lateral etch rate on HOPG to have a pre-exponential factor of $1 \times 10^{10}\left(\mathrm{~s}^{-1}\right)$. Thus $k_{0}{ }^{A S A}$ may be calculated as $2.4 \times 10^{6} \mathrm{~g}$ of reacted carbon per $\mathrm{m}^{2}$ of ASA using Eq. (9). As was done previously, Eq. (14) and Eq. (11) can be combined and rewritten to allow determination of the conversion function from the experimental data as follows: 


$$
f^{A S A}(\alpha)=\frac{m_{0}}{A S A_{0}} \frac{d \alpha}{d t} k_{0}^{A S A} \exp \left(\frac{E_{A}}{R T}\right)
$$

It is theoretically possible to measure all the unknowns in Eq. (15) beforehand to determine the conversion function directly without the need for arbitrary scaling. However, flake graphite has a very low ASA, making it very difficult, at present, to measure this value experimentally. In this case the initial active site density $\left(\Gamma_{0}=A S A_{0} / m_{0}\right)$ may be estimated as the value required to scale the experimental curve to start at one.

\section{Experimental}

A natural flake graphite powder, designated RFL, was obtained from Graphit Kropfmühl AG (Germany). The material was purified by the supplier with an acid treatment and a high-temperature soda ash burn-up to a purity of 99.91 mass $\%$. The as-received RFL material was wet sieved in ethanol to break up any agglomeration and only the fraction between 200 and $250 \mu \mathrm{m}$ was retained, as shown in Fig. 1. All thermal analysis and oxidation was conducted in a TA Instruments SDT Q600 thermogravimetric analyzer (TGA).

Despite this sample being apparently ideal, several stumbling blocks were encountered before reliable kinetic data could be obtained. Initially, powdered samples were placed in $90 \mu \mathrm{L}$ platinum pans. The sample size chosen was as small as possible to avoid possible mass transfer limitations. However, when the sample size was reduced to below $1 \mathrm{mg}$, repeatability became an issue. Since this problem is presumably caused by sample inhomogeneity, it is critical to ensure that the sample is large enough to ensure averaging of the flake irregularities. Thus a sample size of $2 \mathrm{mg}$ was chosen. The largest size fraction obtained during sieving was used. Smaller particles would allow a larger averaging effect, but it was decided that it would be more beneficial to further mitigate possible mass transfer limitations by using large flakes with a low packing density.

Despite these precautions, mass transfer limitations were still found to be present when experiments were conducted at different purge rates which yielded different reaction rates under otherwise identical conditions. A high purge rate of $500 \mathrm{~mL} / \mathrm{min}$ of pure instrument grade (IG) oxygen (>99.999\%) was already being used to keep the gas change-over (from inert to oxidizing during isothermal experiments) as short as possible and limit the possibility of secondary reactions with the reaction products. However, it should be noted that in this reaction the desorption is assumed to occur irreversibly, so it was not expected that the gaseous product would influence the reaction to a large degree. To explain these persistent observations, consider the gas flow across a sample pan for a beam-type TGA, shown in Fig. 5a. 


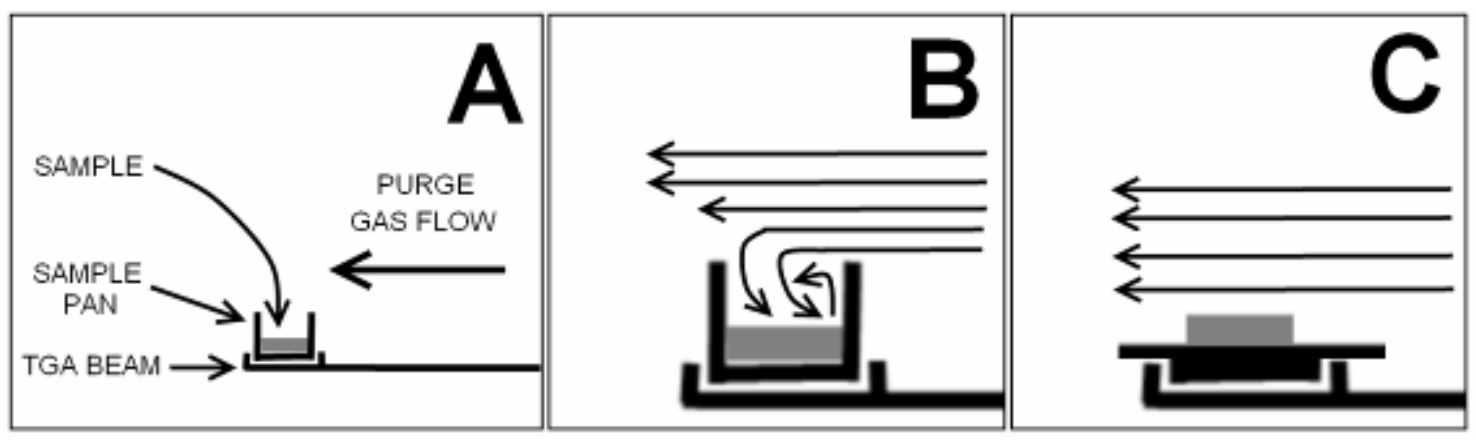

Fig. 5: Experimental gas flow

It is inevitable that eddies will develop in the flow above and inside the pan, as shown in Fig. 5b. These currents may result in the formation of vortices or stagnant zones, which may lead to reactant gas depletion or product gas build-up. Instead, flat platinum discs, with no walls on which the sample powder could be lightly deposited, were used, as indicated schematically in Fig. 5c. The absence of mass transfer limitations for this new configuration was confirmed by varying the purge rate under identical conditions.

For isothermal runs, samples were heated in IG argon (>99.999\%) flowing at $300 \mathrm{~mL} / \mathrm{min}$ at a scan rate of $50^{\circ} \mathrm{C} / \mathrm{min}$ from ambient to between $600^{\circ} \mathrm{C}$ and $800^{\circ} \mathrm{C}$. When the desired reaction temperature was reached, the temperature was allowed to stabilize for 15 minutes and then the argon flow was shut off and IG oxygen flow was started at $500 \mathrm{~mL} / \mathrm{min}$. The experiments were conducted at $600,650,700,750$ and $800{ }^{\circ} \mathrm{C}$. In most cases the experiment was repeated at least three times at a given temperature and the results averaged.

For non-isothermal experiments, the samples were heated in IG oxygen flowing at $500 \mathrm{~mL} / \mathrm{min}$ at heating rates of 3,4 and $5^{\circ} \mathrm{C} / \mathrm{min}$ from ambient to $1000^{\circ} \mathrm{C}$. The experiment was repeated three times for each rate and the data averaged. The purge gas outlet of the TGA was connected to a Pfeiffer QMS 200 mass spectrometer (MS). During an empty control experiment, the dynamics of the gas change from inert to oxidizing were found to be very quick and adequately characterized by a first-order dynamic process with a time constant of $\tau \approx 2.6 \mathrm{~min}$. During oxidizing experiments, the MS showed the presence of only $\mathrm{CO}_{2}$ in the gas product stream.

For both the isothermal and non-isothermal experiments, empty baseline runs were conducted to account for any buoyancy effects. It was found that in addition to these effects, the empty sample holder exhibited drift over time. The manufacturer stated that the baseline drift for this TGA is $0.025 \mathrm{mg} / \mathrm{h}$, close to the observed value of $0.02 \mathrm{mg} / \mathrm{h}$. For the sample size this equates to a $1 \%$ drift over a $1 \mathrm{~h}$ period. For low-temperature experiments, which may last up to $10 \mathrm{~h}$, this would imply a drift of $10 \%$. To minimize this effect, the minimum reaction temperature was chosen to ensure a run length of less than $10 \mathrm{~h}$. In accordance with ICTAC recommendations [5], these baseline signals were subtracted from the experimental data to remove these effects from the data to some extent.

For isothermal experiments, the temperature signals were analysed to ensure that the correct temperature was achieved and maintained. It was found that increasing the purge gas flow to the chosen value prevented any sample temperature excursions during high-temperature runs by effectively removing the heat generated. For non-isothermal experiments, the measured temperature signals were used for all kinetic calculations. Despite the baseline correction, a systematic 
deviation at low conversions was noticed for the isothermal experiments: the measured rate was lower than expected when comparing different runs.

This effect increased as the temperature, and hence the reaction rate, was increased. This is caused by the gas change which takes place over the same time frame for each experiment but, due to the higher reaction rate, persists to higher conversions at higher temperature. The effect can be partially compensated for by assuming a gas reaction order, i.e. $P_{\mathrm{O} 2}{ }^{m}$ where $P_{\mathrm{O} 2}$ is the oxygen partial pressure, and using the first-order time constant mentioned earlier for the gas change from inert to oxidizing. This was done for all the isothermal experiments and a reaction order of $m \approx 0.4$ was found to provide satisfactory compensation.

Extreme care was taken to ensure that reliable, meaningful kinetic data were produced. Furthermore, several isothermal and non-isothermal data sets were generated to ensure consistent results, across a wide temperature range.

\section{Results and discussion}

The averages of the conversion and conversion rate for each of the isothermal run data sets are shown in Fig. 6, with the exception of the $600{ }^{\circ} \mathrm{C}$ data due to the excessively long time involved.

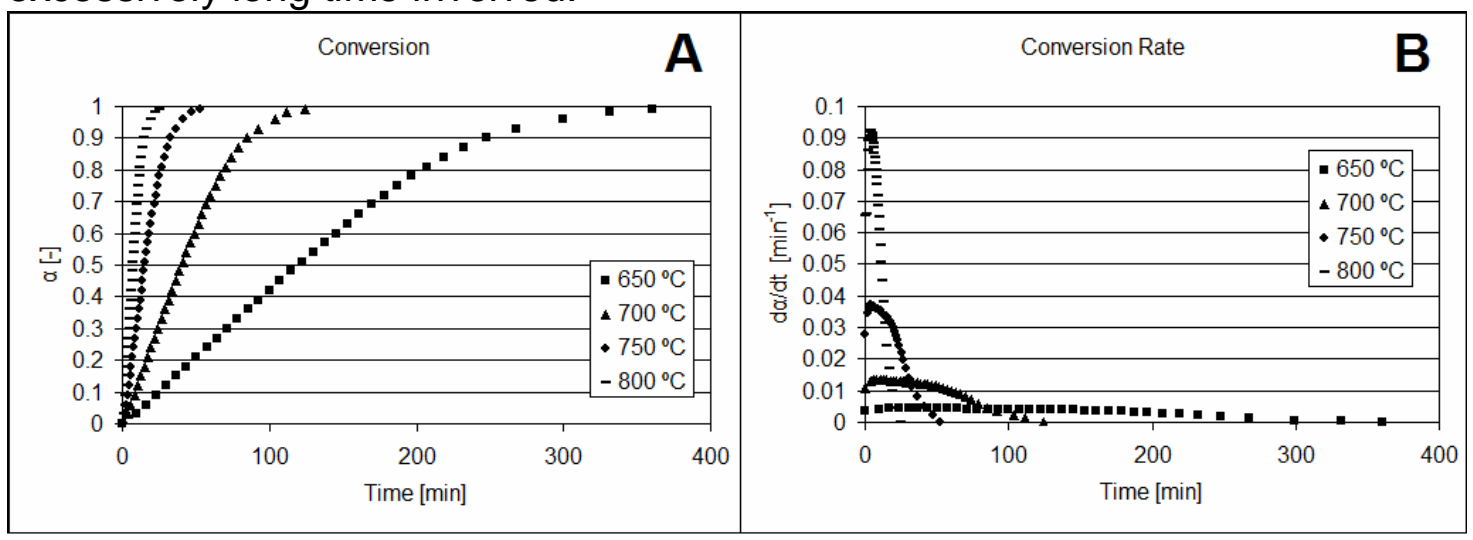

Fig. 6: Conversion and conversion rate for isothermal data sets

The averages of the conversion and conversion rate for each of the nonisothermal run data sets are shown in Fig. 7.

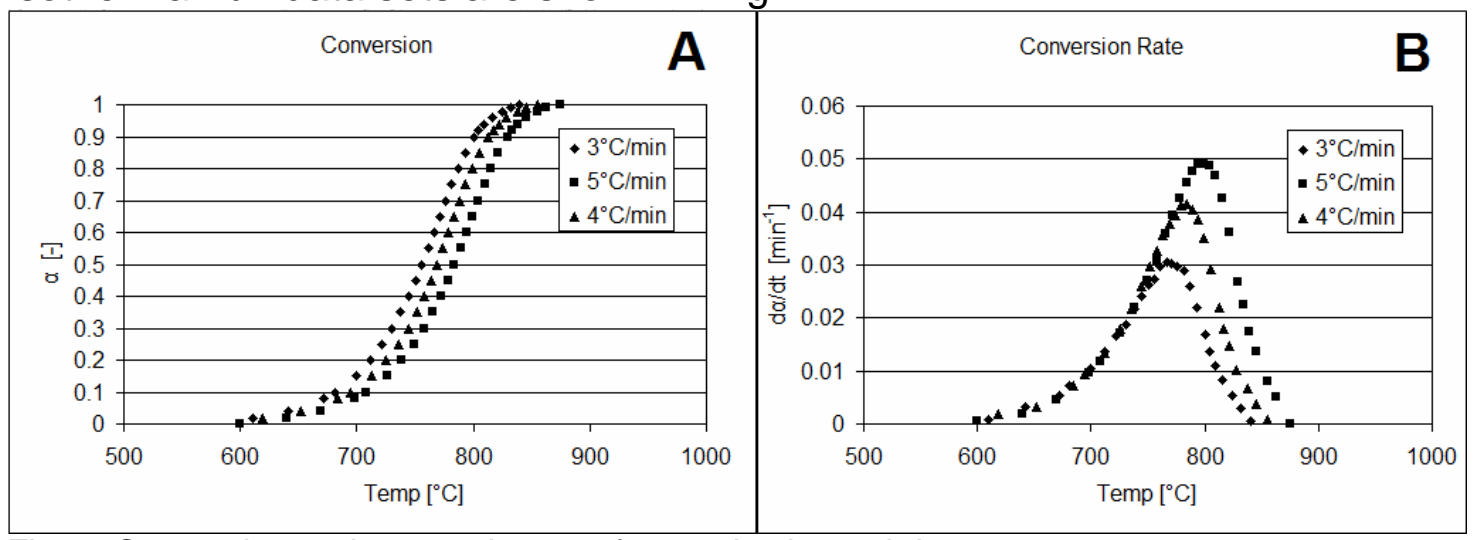

Fig. 7: Conversion and conversion rate for non-isothermal data sets 
The differential isoconversional method recommended by ICTAC [5] for estimating the activation energy was used for both the isothermal and non-isothermal data sets to generate the activation energy as a function of conversion, as shown in Fig. 8.

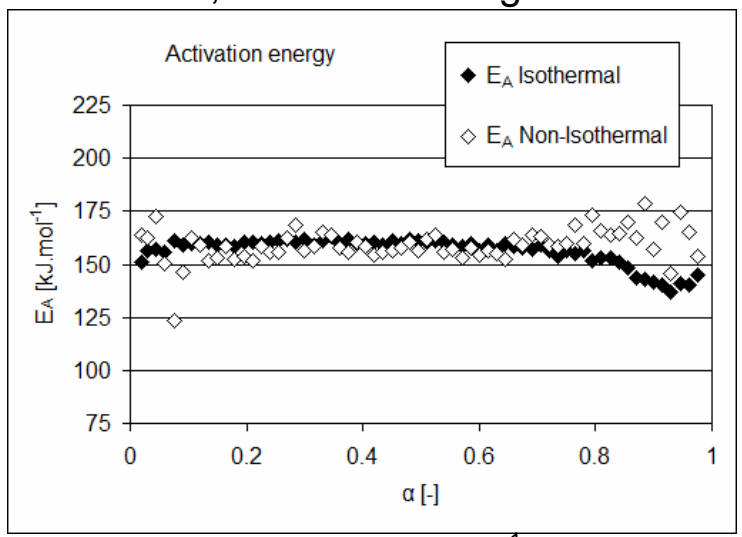

Fig. 8: Activation energy (kJ.mol ${ }^{-1}$ ) estimated for both data sets

The non-isothermal estimate shows a significantly increased level of scatter, especially at high and low conversions. The isothermal data also show a deviation at high conversions. It is not unexpected to see deviations from a constant activation energy at these regions of conversion. Firstly, the flakes are not perfectly regular and contain some erratic edge regions. These areas represent a local active surface area density that is higher than average and that will be reacted away more rapidly until a steady state roughness is achieved. This effect will not be consistent across all samples and can explain the deviation at low conversions. This irregularity will again come into play at high conversions where the differences in the original geometries become most pronounced. Flakes will now start to break apart as any structural irregularities overwhelm the original macro flake structure. However, based on the wide range of conversions with reasonably consistent values and the agreement of the isothermal and non-isothermal measurements, it is possible to state with a fair degree of certainty that the true activation energy has been determined.

The activation energy was found to be $157.7 \pm 4.2 \mathrm{~kJ} \mathrm{~mol}^{-1}$. This is the average of all the isothermal and non-isothermal data sets across the entire range of conversion. Hahn [11] found the activation energy for this reaction to be $143 \mathrm{~kJ}^{\mathrm{mol}}{ }^{-1}$ which is comparable to the experimentally determined value.

Using the classic approach, the reaction rate data can now be scaled to obtain the normalized conversion function with a range of between zero and one. The values of $k_{0}$ to achieve this were found to be 70,000 for the isothermal data and 54,000 for the non-isothermal data. The reaction model curves are shown in Fig. 9, together with the normalized model of an ideal disc. 


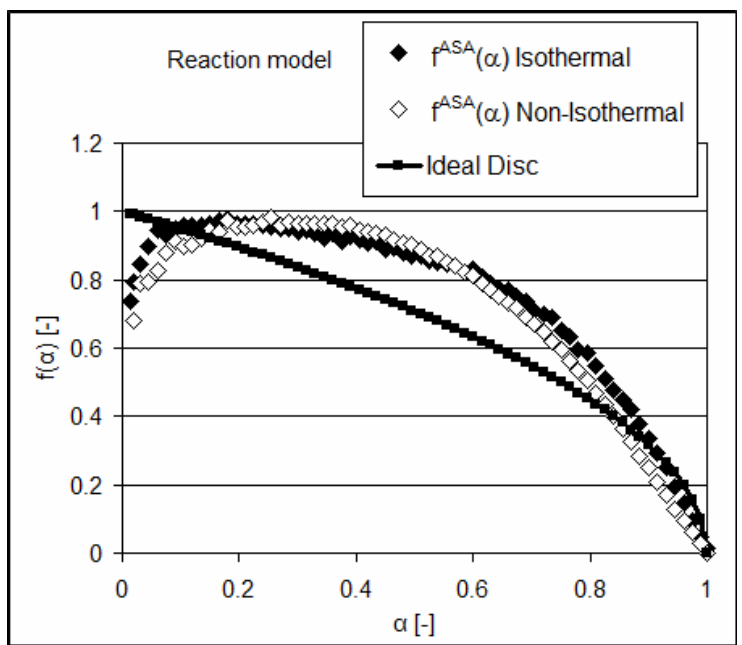

Fig. 9: Reaction model curves for both data sets and the ideal disc

As can be seen from Fig. 9, the conversion behaviour does not conform to that of the ideal disc. It is not clear why the initial value for the graphite should be around 0.7 as opposed to the starting value of the ideal disc which is one. This makes establishing the relationship between the two curves problematic. In fact, from Fig. 9 it is not readily apparent that they are even related.

Indeed, the experimental model does not conform to any of the common reaction models [5]. The Avrami-Erofeev model exhibits vaguely similar behaviour since it also proceeds through a maximum. However, mathematically, the Avrami-Erofeev model must strictly begin and end at a value of zero. Although it may be possible to pick a larger value for $k_{0}$ to bring the starting value close to zero, this would reduce the peak value to below one. Such a scaling would be even more illogical since it is highly doubtful that nucleation and growth is the correct mechanism for this reaction.

This demonstrates the problem with using an arbitrary scaling procedure. From visual inspection and theoretical knowledge it seems certain that the reaction of graphite with oxygen should be governed by the progression of the reaction interface, but based on Fig. 9 alone it is difficult to deduce the relationship, if any, between the ideal model and the experimental curve. The situation is further exacerbated if one tries to relate the experimental behaviour to one of the other reaction mechanisms, since the link becomes even more unsubstantiated.

The integrated expression given by Pijolat et al. [10] in Eq. (6) represents an initial step towards reconciling all the solid state kinetic reactions into a single comprehensive scheme. The expression given by Eq. (11) represents a generalized subset of reactions where reactivity is controlled by the reaction interface alone. The conversion function shown in Fig. 9 is difficult to interpret without the clear link to the fundamental reactivity that Eq. (11) provides.

To demonstrate this, the initial active site density can be determined, as mentioned earlier, by choosing a value that scales the conversion function models to start at one, in conjunction with the experimental activation energy and the other values as determined from the literature. This was found to be a value of $\Gamma_{0}=0.015$ $\mathrm{m}^{2} / \mathrm{g}$ for the non-isothermal data. A small discrepancy exists for the iso-thermal value. This is presumably caused by inadequate compensation for the gas changeover, i.e. the initial change from inert to oxidizing. Based on visual observation, the sample may be considered to be a collection of perfect discs with a radius of $230 \mu \mathrm{m}$ having a crystal density of $\rho=2.26 \mathrm{~g} / \mathrm{cm}^{3}$ [4], which means that the initial active surface area density may be estimated as $\Gamma_{0}=0.008 \mathrm{~m}^{2} / \mathrm{g}$. This is reasonably close 
to the experimentally determined value, despite the assumptions inherent in the estimation of $k_{0}^{A S A}$. The revised ASA conversion function curves are given in Fig. 10.

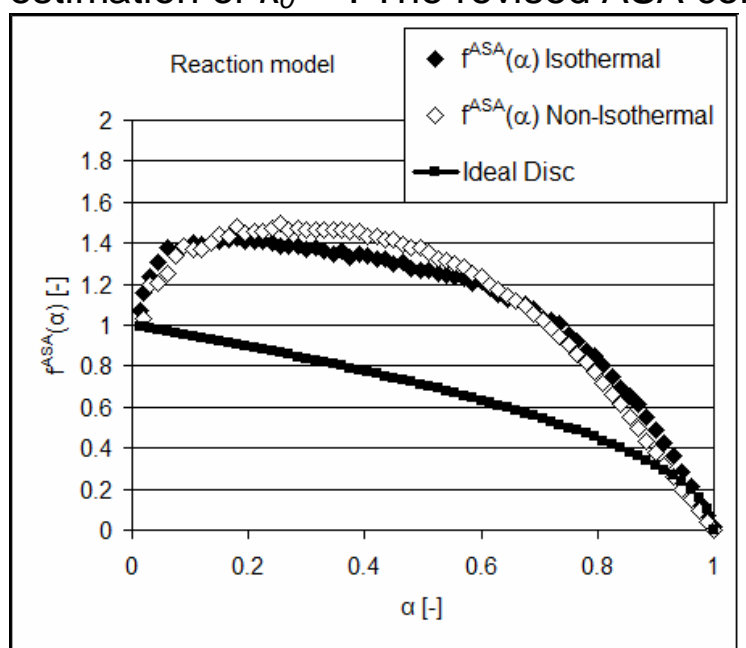

Fig. 10: ASA conversion function models

Analysis of this curve shows that it is quite different from the previous conversion function shown in Fig. 9. It has a clear physical interpretation: initially, up to conversions of between 20 and $40 \%$, the active surface area increases, beyond which it declines to zero. When the microstructure of an oxidized natural graphite flake is examined under the SEM, as shown in Fig. 11a and b, some interesting characteristics are apparent.

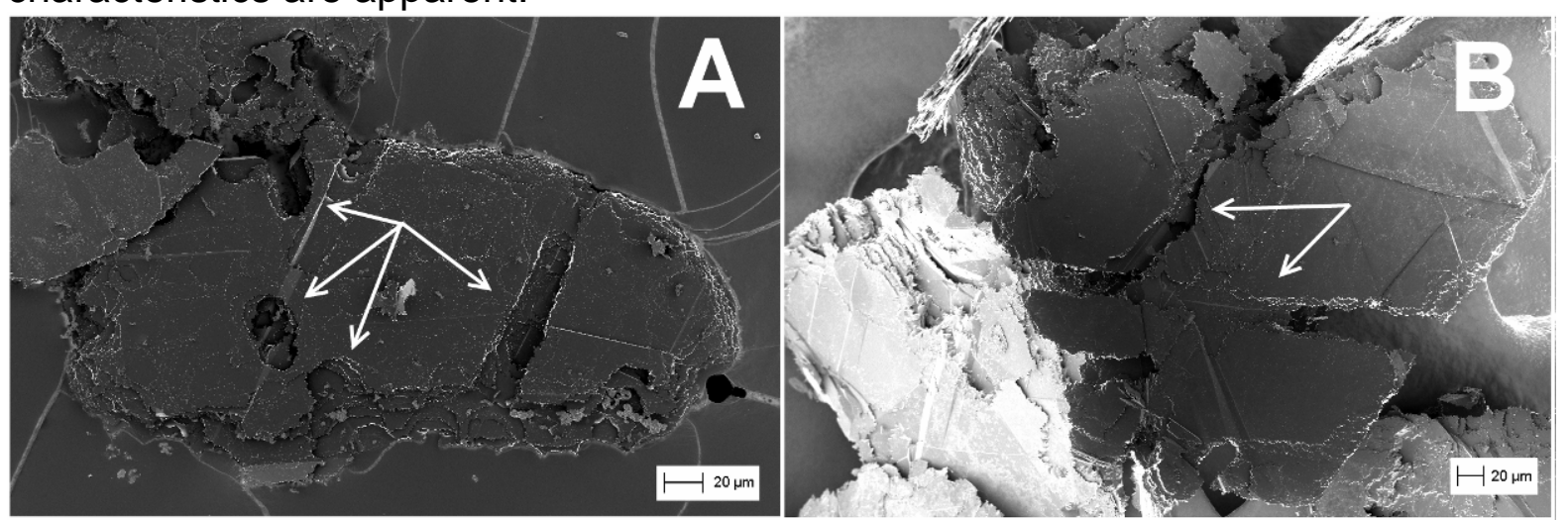

Fig. 11: SEM images of oxidized microstructure

During oxidation, defects within the macro flake structure become evident. They include, among others, large fissures stretching across the flake and the development of circular pits. These are presumably caused by crystal defects or pitting impurities, or some combination of both. As oxidation proceeds, these defects grow, creating additional reaction interface from which gasification proceeds, despite the shrinking macro flake body. Eventually the defects coalesce, decreasing the ASA and reaction rate. This is consistent with the expectations from the new ASA conversion function and indicates why the behaviour differs from that of an ideal disc and all of the other geometrical models, which are monotonically decreasing. Hence it is clear that they both have the same initial ASA and that the ASA of the graphite increases relative to that of a simple disc due to the growth of these defect regions. It also demonstrates the reason for the vague correspondence to the nucleation and growth model. 
In a previous publication the present authors explored the analytical expressions for a variety of different geometrical ASA developments [13]. However, it is very difficult to derive analytical expressions for the geometrical progressions found in real flakes since the defect regions are randomly distributed throughout the macro flake structure. It is clear that the definition of a "characteristic dimension" for such random structures is impossible. Consider the two possible representative geometries shown in Fig. 12.

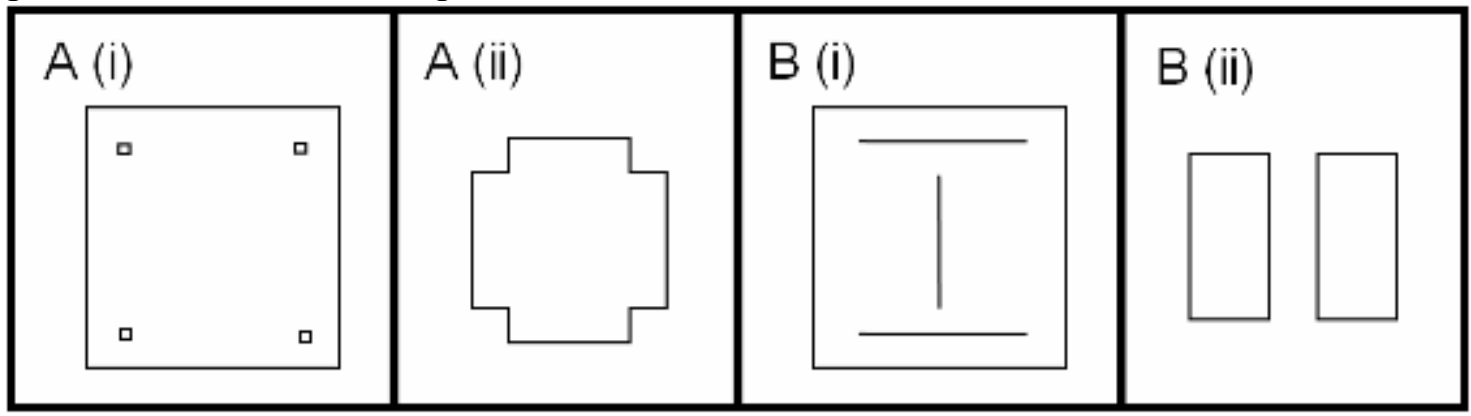

Fig. 12: Possible reaction models

Model A represents four, infinitesimal, square holes placed close to the edge of a square disc. Model B represents a square disc with three infinitesimal fissures cut into the structure. The conversion functions for these models are compared with those for the ASA conversion model for RFL graphite, shown as an average of isothermal and non-isothermal data in Fig. 13.

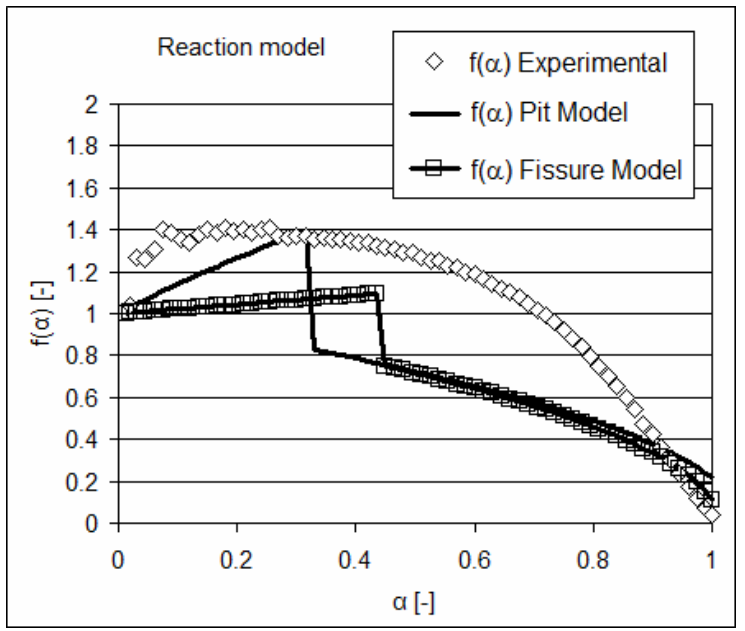

Fig. 13: Theoretical ASA conversion functions

Several two-branch fissure structures were considered, but none was found to be capable of producing an increasing conversion function. This is understandable if one considers that a single fissure can at best counteract the ASA loss from two shrinking peripheral surfaces. Only the structure shown with three fissures provides a net gain in ASA. From visual inspection it is unlikely that three such fissures will be found growing independently inside a single flake. Thus it is more likely that the fissures lead to fragmentation effects, while the pits lead to a more pronounced active surface area creation - hence the observed behaviour.

The analytical models are only capable of semi-qualitatively representing the observed behaviour. Both models show a growth in ASA until the point where coalescence of the substructures occurs, beyond which they undergo a decline similar to that of the standard disc. These models, however, suffer from two clear shortcomings. Firstly, they both undergo a discontinuity in ASA development. This is 
caused by a transition to a secondary state; these states are indicated by Fig. 12 $\mathrm{A}$ (ii) and Fig. $12 \mathrm{~B}$ (ii). They reflect the instantaneous loss of interfacial area as the substructures coalesce and the secondary structures take over. Since the real flakes contain distributions of defects at random positions in the flake, such transitions would be averaged, leading to a much smoother behaviour.

Secondly, something which is not clearly visible in Fig. 13, these models tend towards a non-zero value when conversion tends towards $100 \%$. For real structures undergoing reaction at an interface this is not possible since the rate will decline gradually as the remaining material is reacted away. Again this is caused by the instantaneous loss of large interfacial areas. This is easily visualized for Fig. 12 B(ii), where the rectangles would shrink until only elongated slivers of material were left. These entire slivers would disappear in a single instant, although the overall rate would still be quite high. The longer the sliver, the more pronounced the offset from zero. Ultimately, for an infinite plate the rate would be constant across all conversions.

Thus these models do not provide an adequate representation of the observed behaviours. They do, however, demonstrate that the experimental conversion functions can be qualitatively linked to observable microstructural development taking place within the flakes.

The link between the two models proposed in Fig. 12, the experimentally obtained conversion functions and the ideal disc model would not have been possible if all the conversion functions did not start at one. It is clear that if scaled to vary strictly between zero and one, all four conversion functions would have different starting values. In this case it would be impossible to distinguish between the effect of initial ASA differences and the intrinsic reactivity.

\section{Conclusions}

The ICTAC-recommended [5] approach was used to characterize the gasification of high-purity, highly crystalline, large natural graphite flakes in oxygen. The differential isoconversional method was followed to derive the activation energy for the reaction, which remains fairly constant over a wide range of conversions. The average activation energy was found to be $157.7 \pm 4.2 \mathrm{~kJ}_{\mathrm{mol}}{ }^{-1}$. Extreme care was taken to ensure that the kinetic data collected were reliable and consistent, using both isothermal and non-isothermal data sets over a wide temperature range.

The conversion function for this reaction was determined in the classic way by selecting an appropriate value of the pre-exponential constant to scale the reaction rate curve to vary between zero and one. Comparison of the obtained conversion function with commonly established reaction models did not allow identification of the mechanism governing the oxidation.

Based on the graphite properties and the simple gasification reaction taking place, it was expected that the conversion function would be that of a shrinking disc. However, the observed conversion function exhibited an increase from a non-zero initial value to a maximum between 20 and $40 \%$ conversion, followed by a decline to zero, vaguely resembling nucleation and growth. Due to the arbitrary scaling of the conversion function it is difficult to decouple the relationship between the reactivity and the ASA or geometry and hence to compare or link different and especially new reaction models. 
Due to these and other shortcomings a more formalized integrated approach to solid-gas reactions has been proposed by Pijolat et al. [10]. Their equation represents an initial step towards reconciling all the solid state kinetic reactions into a single comprehensive scheme. A generalized expression is derived from fundamental considerations for the subset of reactions where reactivity is controlled by the reaction interface alone. This expression represents a specific case of the equation by Pijolat et al. [10] but a generalization of the classic solid state kinetic expressions.

The expression yields a scaled conversion function that always starts at one but theoretically contains no unknown terms that need to be chosen arbitrarily. Due to the very low ASA of graphite it is very difficult to measure in practice. Instead, the value was estimated from the experimental data, yielding a number that closely corresponds to the calculated value for a collection of discs.

Visual observations of the oxidized flakes indicate the growth of defect structures within the macro flake. These create additional active surface area for reaction, increasing the rate. Eventually these defects coalesce, decreasing the ASA and the reaction rate. Overall, this resembles nucleation and growth behaviour, despite being a reaction interface-controlled reaction. In the context of these observations and a starting value of one, the observed conversion function makes physical sense and the behaviour can be linked to, but distinguished from, the shrinking disc. The complexity and random nature of the oxidized flake microstructure makes it difficult to develop representative analytical models for this behaviour.

Thus this generalized approach provides a fundamental basis for comparing very different geometrical ASA progressions for solids undergoing interface-controlled reaction. This represents a consistent starting point for evaluating different reaction models in this category. Furthermore, this investigation points to the necessity of moving away from the classic approach to a more formalized, generally applicable expression such as that proposed by Pijolat et al. [10] (Eq. (6)).

\section{Acknowledgments}

This work is based on research supported by the Skye Foundation and the South African Research Chairs Initiative (SARChi) of the Department of Science and Technology (DST) and the National Research Foundation (NRF). Any opinion, findings and conclusions or recommendations expressed in this material are those of the authors and therefore Skye, the NRF and the DST do not accept any liability with regard thereto.

\section{References}

[1] A.K. Galwey, M.E. Brown, Thermal Decomposition of Ionic Solids, Elsevier, Amsterdam, 1999.

[2] E.G. Prout, F.C. Tompkins, Thermal decomposition of potassium permanganate, Trans. Faraday Soc. 40 (1944) 488-498.

[3] M. Wissler, Graphite and carbon powders for electrochemical applications, J. Power Sources 156 (2006) 142-150.

[4] H.O. Pierson, Handbook of Carbon, Graphite, Diamond and Fullerenes.

Properties, Processing and Applications, Noyes Publications, New Jersey, 1993. 
[5] S. Vyazovkin, A.K. Burnham, J.M. Criado, L.A. Pérez-Maqueda, C. Popescu, N. Sbirrazzouli, ICTAC Kinetics Committee recommendations for performing kinetic computations on thermal analysis data, Thermochim. Acta 520 (2011) 1-19.

[6] P.L. Walker Jr., R.L. Taylor, J.M. Ranish, An update on the carbon-oxygen reaction, Carbon 29 (1991) 411-421.

[7] J.M. Thomas, E.E.G. Hughes, Localized oxidation rates on graphite surfaces by optical microscopy. Carbon 1 (1964) 209-214.

[8] A. Khawam, D.R. Flanagan, Solid-state kinetic models: Basics and mathematical fundamentals, J. Phys. Chem. B 110 (2006) 17315-17328.

[9] R.H. Hurt, B.S. Haynes, On the origin of power-law kinetics in carbon oxidation, Proc. Comb. Inst. 30 (2005) 2161-2168.

[10] M. Pijolat, L. Favergeon, M. Soustelle, From the drawbacks of the Arrhenius-f( $\alpha)$ rate equation towards a more general formalism and new models for the kinetic analysis of solid-gas reactions, Thermochim. Acta 525 (2011) 93-102.

[11] J.R. Hahn, Kinetic study of graphite oxidation along two lattice directions, Carbon 43 (2005) 1506-1511.

[12] N.R. Laine, F.J. Vastola, P.L. Walker Jr., Importance of active surface area in the carbon-oxygen reaction, J. Phys. Chem. 67 (1963) 2030-2034.

[13] H. Badenhorst, W.W. Focke, Geometric effects control isothermal oxidation of graphite flakes, J. Therm. Anal. Calorim. 108 (2012) 1141-1150.

\section{Appendix A}

Consider the expressions for a disc and a sphere:

$$
\begin{array}{ll}
\text { ASA }=2 \pi r d & \text { ASA }=4 \pi r^{2} \\
\text { mass }=\rho \pi r^{2} d & \text { mass }=\frac{4}{3} \pi \rho r^{3}
\end{array}
$$

These shapes both represent a very specific case where:

$$
\begin{array}{rlrl}
\frac{d m}{d t} & =2 \pi r d \rho \frac{d r}{d t}=\rho A S A \frac{d r}{d t} & & \text { (disc) } \\
\frac{d m}{d t}=4 \pi r^{2} \rho \frac{d r}{d t}=\rho A S A \frac{d r}{d t} & & \text { (sphere) }
\end{array}
$$

Thus, in general, for these shapes:

$$
\frac{d m}{d t}=\rho A S A \frac{d r}{d t}
$$

Both these shapes can be represented by the generalized expression: mass $=\rho r^{n} f_{0}$ where $f_{0}=\pi \mathrm{d}$ for a disc and $4 \pi / 3$ for a sphere and $\mathrm{n}=2$ or 3 respectively. Since

$$
\frac{d m}{d t}=\rho A S A \frac{d r}{d t}=\rho n r^{n-1} f_{0} \frac{d r}{d t}
$$

Thus

$$
A S A=n r^{n-1} f_{0}
$$

But 
$\frac{d \alpha}{d t}=\frac{-1}{m_{0}} \frac{d m}{d t}=\frac{-\rho z r^{n-1} f_{0}}{\rho r_{0}^{n} f_{0}} \frac{d r}{d t}=\frac{-n}{r_{0}{ }^{n}} r^{n-1} \frac{d r}{d t}$

Since $r=r_{0}(1-\alpha)^{1 / n}$ starting from $m=m_{0}(1-\alpha)$

$r^{n-1}=\frac{r^{n}}{r}=\frac{r_{0}^{n}}{r_{0}}(1-\alpha)^{n-1 / n}$

Thus

$\frac{d \alpha}{d t}=-\frac{n}{r_{0}}(1-\alpha)^{n-1 / n} \frac{d r}{d t}$

It is very important to note that up to this point two assumptions have been made:

- The particle mass, at any time during the reaction, can be expressed in terms of a single "characteristic dimension".

- The change in mass of the particle, i.e. the mass of material reacted, is linearly proportional to the surface area undergoing reaction and the change in the characteristic dimension.

If one now makes a final assumption: $r=r_{0}-v t$

Then

$\frac{d r}{d t}=-v$

And thus

$\frac{d \alpha}{d t}=\frac{v}{r_{0}} n(1-\alpha)^{n-1 / n}$

Which is the classic solid state kinetic expression with

$\frac{d \alpha}{d t}=k(T) f(\alpha)$

where

$f(\alpha)=n(1-\alpha)^{n-1 / n}$

$k(T)=\frac{v}{r_{0}}$

However, now compare this to the function derived in the text, i.e. Eq. (9)

$\frac{d \alpha}{d t}=\frac{A S A_{0}}{m_{0}} k^{A S A}(T) f^{A S A}(\alpha)$

where

$f^{A S A}(\alpha)=A S A / A S A_{0}$

But

mass $=\rho r^{n} f_{0}$

and

$A S A=n r^{n-1} f_{0}$

Thus we have:

$\frac{d \alpha}{d t}=k_{A S A}(T) \frac{n r^{n-1} f_{0}}{\rho r_{0}{ }^{n} f_{0}}=\frac{k_{A S A}(T)}{\rho} \frac{1}{r_{0}} n(1-\alpha)^{n-1 / n}$

Since 
$r^{n-1}=\frac{r^{n}}{r}=\frac{r_{0}^{n}}{r_{0}}(1-\alpha)^{n-1 / n}$

Thus with $k_{A S A}(T)=\rho v=\rho r_{0} k(T)$ the two expressions (A1 and A2) are identical and equivalent. Furthermore, consider

$f^{A S A}(\alpha)=\frac{A S A}{A S A_{0}}=\frac{n r^{n-1} f_{0}}{n r_{0}^{n-1} f_{0}}=(1-\alpha)^{n-1 / n}$

Hence:

$f^{A S A}(\alpha)=\frac{1}{n} f(\alpha)$ 